\title{
Oral Histoplasmosis: Report of Two Cases
}

\author{
SMA SADAT ${ }^{\mathrm{a}}$, SN RITA ${ }^{\mathrm{b}}$, MA KAHHAR $^{\mathrm{c}}$
}

Summary:
Histoplasmosis is a deep fungal infection maypresent as oral
ulceration and can be confused with oral mucosal
malignancies, tuberculosis and other fungal diseases. It
mostly affects individual having immunodeficiencies like
tuberculosis and AIDS, but may also affect immunocompetent
individuals. Here we have presented two cases of such oral

Introduction:

Histoplasmosis is a granular mycosis caused by Histoplasma capsulatum, a dimorphic pathogenic fungus that develops a branching hyphal form in soil and a yeast form in the host tissue ${ }^{1}$. Infection by this organism is endemic in North America, Central America and South America, but it also has been reported in Africa, Asia, India and Australia ${ }^{2}$. The infective agents are airborne spores from the mycelial form. In Bangladesh, sporadic cases have been reported in different literature ${ }^{3-6}$.The inhalation of spores, which are present in chicken, pigeon and bat excrement, by immunocompetent hosts usually results in an asymptomatic pulmonary infection. Hosts with immunosuppression may develop a generalized infection that involves several organs ${ }^{7}$. Clinically the disease is manifested as weakness, fever, weight loss, mucocutaneous lesions and hepatosplenomegaly.

Histoplasmosis is clinically classified into primary acute pulmonary form, chronic pulmonary form and disseminated form (DH). The course of this disease can be affected by the immune status of the host and by the degree of exposure to infective propagules ${ }^{8}$. Oral lesions

a. Dr. S. M. Anwar Sadat, BDS, MCPS, FCPS, MS (OMS), Resident Surgeon, Oral \& Maxillofacial Surgery, Dhaka Dental College \& Hospital, Dhaka, Bangladesh.

b. Dr. Sufia Nasrin Rita, BDS, FCPS, Assistant Professor and Head, Dept of Orthodontics, Sapporo Dental College, Dhaka, Bangladesh.

c. Professor M. A. Kahhar, MBBS, FCPS, FRCP, Dept of Medicine, Dhaka Medical College \& Hospital, Dhaka, Bangladesh.

Address of Correspondence: Dr. S. M. Anwar Sadat, BDS, MCPS, FCPS, MS (OMS), Resident Surgeon, Oral \& Maxillofacial Surgery, Dhaka Dental College \& Hospital, Dhaka, Bangladesh. Email: an_sadat@yahoo.com. Mobile: 01711156023

Received: 26 February, $2012 \quad$ Accepted: 13 September, 2012 histoplasmosis treated by oral itraconazoles. Complete disappearance of oral ulcerations was evident following three and four weeks treatment respectively and the patients are in regular follow up and maintenance therapy.

Keywords: Histoplasmosis, Oral Ulcer, Itraconazole, PAS

(J Bangladesh Coll Phys Surg 2012; 30: 229-233)

have been reported in 25 to 45 percent of patients with documented cases of progressive disseminated histoplasmosis and in several case reports?

About $30-66 \%$ of patients with DH have oral lesions, frequently presenting as the initial sign. Generally lesions of histoplasmosis in the oral cavity are the local manifestation of pulmonary or disseminated disease $\mathrm{e}^{10}$ but rarely may they be the primary or even the only manifestation of the disease ${ }^{11}$.

In endemic areas, H. capsulatum is a major opportunistic pathogen in patients infected with the human immunodeficiency virus (HIV); approximately 5 percent of patients with AIDS in these areas develop disseminated histoplasmosis ${ }^{12}$. In immunocompetent hosts, few cases of oral histoplasmosis without detectable systemic involvement have been described. Oral histoplasmosis may manifest at any region of the mouth as nodules, deep ulcerative or vegetative lesions or painful shallow lesions ${ }^{13}$.

The diagnosis is usually based on clinical signs and symptoms, organ function tests and fungal demonstration or culture from a lesion or secretion. Serological testing may help together with other suggestive but non-diagnostic clinical criteria ${ }^{14,15}$. Recently, molecular typing of $\mathrm{H}$. capsulatum has been shown to be useful in distinguishing relapse from reinfection, and in defining the likely source of the infection ${ }^{16}$. Furthermore, molecular typing of $\mathrm{H}$. capsulatum by the random amplified polymorphic DNA polymerase chain reaction (RAPD-PCR) method is able to discriminate among clinical isolates, making it a useful tool for epidemiological investigation ${ }^{16}$.

Historically, amphotericin B has been the drug of choice for systemic histoplasmosis ${ }^{17}, 18$. The options have broadened considerably since the introduction of the azole compounds, ketoconazole and itraconazole ${ }^{14,17}$. The successfully treated 1st reported case in Bangladesh was in $1982^{3}$. 


\section{Report of Case - 1:}

A 42-year-old male professionally painter reported with a chief complaint of painful ulcer on whole palatal mucosa and gingiva associated with burning sensation, dysphagia and poor general health for 20 days. Patient also gave history of diarrhoea 5 days following onset of lesion. There was a history of bleeding from the ulcer with difficulty in speech, mastication with no history of pus discharge, paresthesia or numbness. Patient was habituated with smoking and gave no history of extramarital sexual exposure or close contact with known tuberculosis patients. The medical histories of patient were noncontributory.

Extraoral examination revealed imaciated face with mild anaemia and enlarged, firm, non-tender, non-fixed lymph nodes in bilateral submandibular regions. Intraoral examination revealed widespread ulcer in marginal, interdental and attached gingivae, muosa of whole hard palate and also labio buccal gingiva of left maxilla. Granular white patches were evident to the soft palatal mucosa. The ulcers were tender with edematous, raised margin, granular surfaced and easily bleeded on touch. The surfaces were covered with slough and focal areas of necrosis (Figure 1).

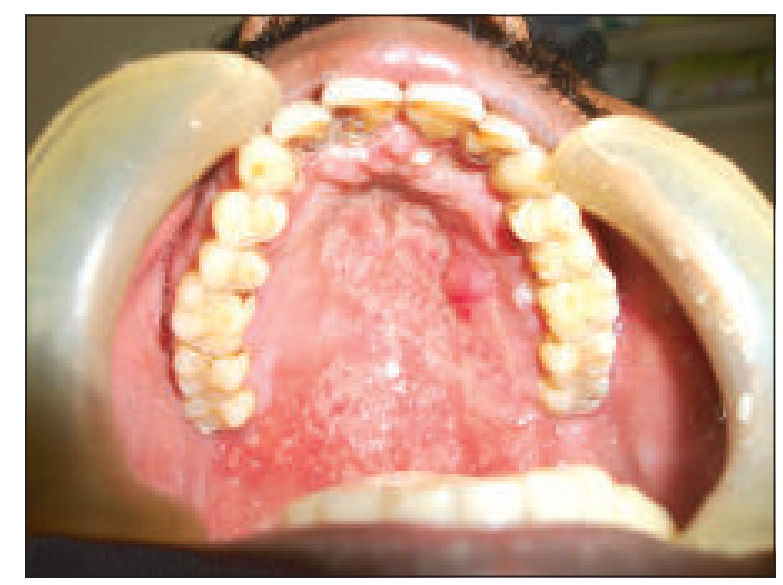

Fig.-1: Ulcer present on the palatal mucosa and gingiva (Case-1)

Haematological values were within normal limits with normal blood sugar level and non-reactive VDRL, HIV1 and HIV2 in Western blot assay. Orthopantomogram showed no significant alvolar bone loss. A chest radiograph did not reveal any abnormality. Initially Nystatin was advised to use locally which caused disappearance of white granular patches from soft palate and part of hard palate. Considering the possibilities of deep fungal infection, tuberculosis or mucosal malignancies, incision biopsy was done which revealed ill defined granulomata; tiny round and oval bodies, morphologically consistent with histoplasmosis. There was no evidence of caseous necrosis or malignancy in the tissue sections (Figure 2). Based on the clinical features and investigatory findings, a final diagnosis of oral histoplasmosis in a non-HIV patient was made. Then the patient was evaluated systemically, where systemic involvement of histoplasmosis was ruled out.

With proper education and counseling, patient was treated with oral Itraconozole $200 \mathrm{mg}$ twice daily. The lesions completely healed following three weeks medication (Figure 3). The patient, however, is under regular follow-up with maintenance therapy and no recurrence is noted after two months.

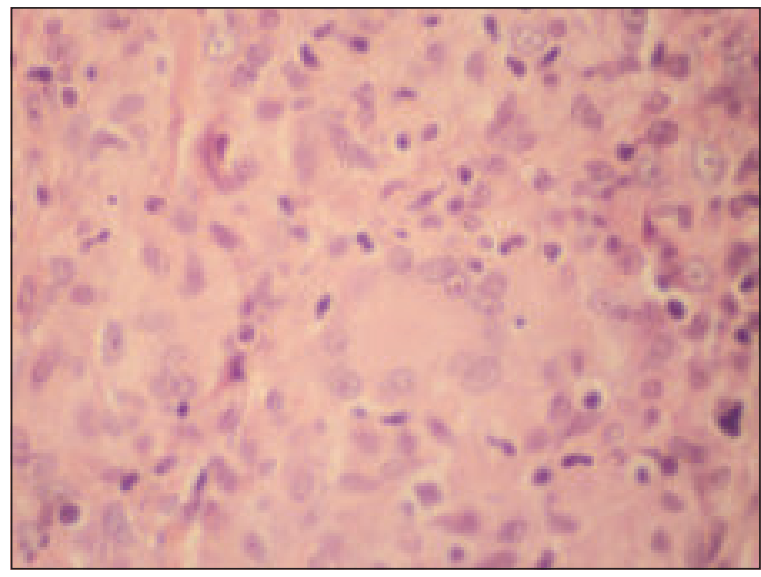

Fig.-2: Histopathology shows epithelioid granulomata with round and oval bodies (Case-1)

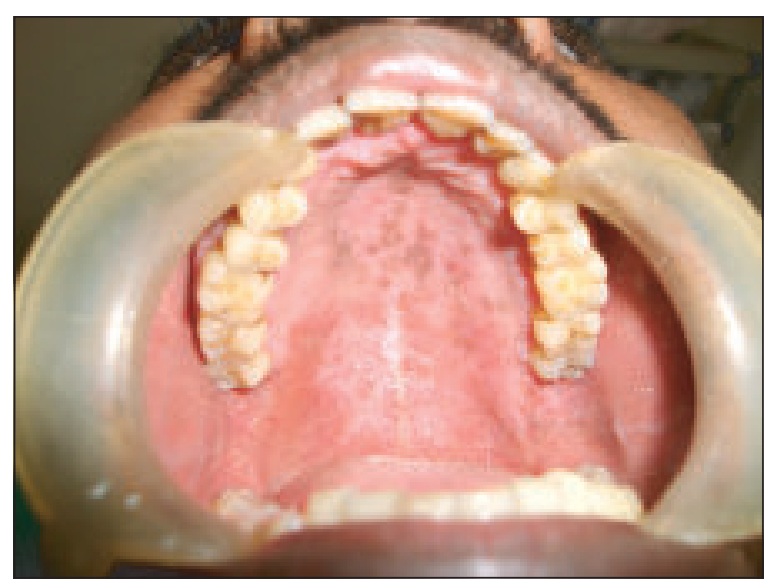

Fig.-3: Post treatment showing complete resolution of palatal lesion (Case-1) 


\section{Report of Case-2:}

A 65-year-old male farmer presented with two painful ulcers on upper alveolar gingivae and palatal mucosae and poor general health for three weeks. There was a history of difficulty in mastication with no history of pus discharge, paresthesia or numbness. Patient was habituated with smoking and gave no history of extramarital sexual exposure. He gave history of previous pulmonary tuberculosis with irregular treatment. The other medical histories of patient were noncontributory.

Extraoral examination revealed imaciated face with mild anaemia and enlarged, firm, non-tender, non-fixed lymph nodes in bilateral submandibular regions. Intraoral examination revealed poor oral hygiene with two almost identical ulcers in maxillary alveolar ridge and palatal mucosae on both sides. The indurated ulcers were tender with edematous, raised margin and the surfaces were covered with slough (Figure 4).

Haematological values were within normal limits with normal blood sugar level and non-reactive VDRL, HIV1 and HIV2 in Western blot assay. Related investigations were done to exclude dissemination of the disease. Orthopantomogram showed no significant alvolar bone loss. Considering the possibilities of deep fungal infection, tuberculosis or mucosal malignancies, incision biopsy was done. Initial biopsy report revealed severely inflammed and oedematous fibrocollagenous tissue covered by ulcerated stratified squamous epithelium showing minor salivary glands with multiple granulomata composed of epitheloid cells with extensive areas of caseation necrosis which was consistent with tuberculosis. The patient was reevaluated by a group of physicians who recommended repeat biopsy which revealed dense infiltration of acute and chronic inflammatory cells including many eosinophils, aggregates of epitheloid histiocytes and some of the histiocytes contained intra-cytoplasmic round to oval bodies; morphologically consistent with histoplasmosis. There was no evidence of caseous necrosis or malignancy in the tissue sections (Figure 5 ). Based on the clinical features and investigatory findings, a final diagnosis of oral histoplasmosis in a non-HIV patient was made. Then the patient was evaluated systemically, where systemic involvement of histoplasmosis was ruled out.

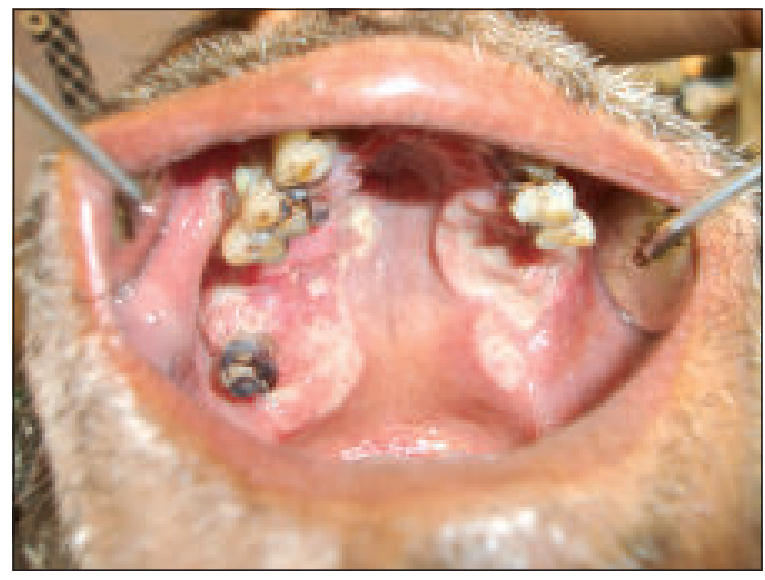

Fig.-4: Ulcer present on the palatal mucosa and gingiva (Case-2).

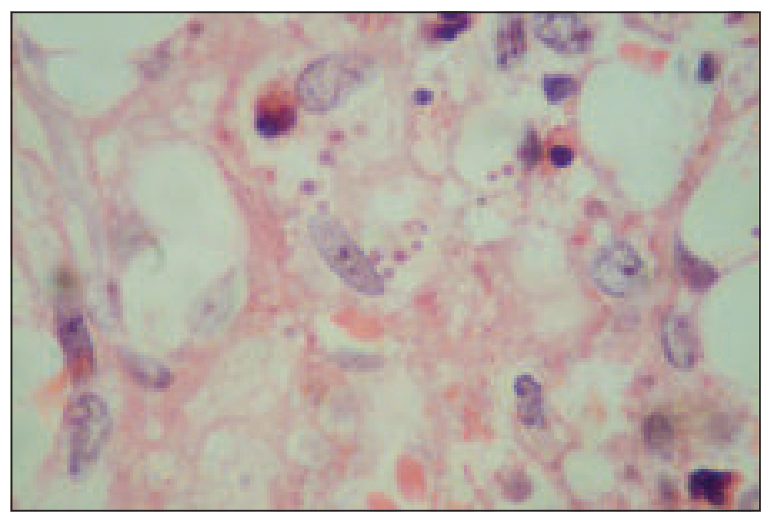

Fig.-5: Histopathology shows epitheloid histiocytes with intracytoplasmic round to oval bodies (Case-2)

With proper education and counseling, patient was treated with oral Itraconozole $200 \mathrm{mg}$ twice daily. The lesions completely healed following four weeks medication (Figure 6). The patient, however, is under

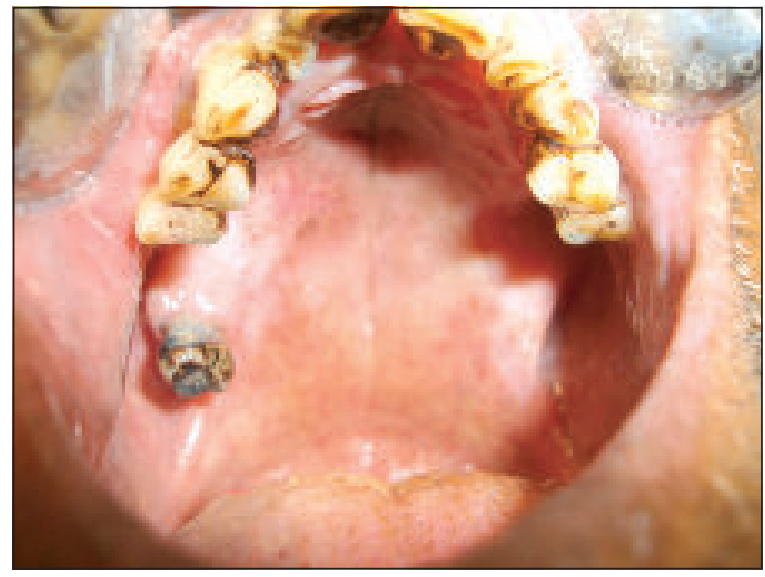

Fig.-6: Post treatment showing complete resolution of palatal lesion (Case-2) 
regular follow-up with maintenance therapy of Itraconazole $200 \mathrm{mg}$ once daily and no recurrence is noted after two months.

\section{Discussion:}

H. capsulatum is the organism responsible for occuring the fungal infection, Histoplasmosis. Oral manifestations, usually associated with the chronic disseminated form of the disease, constitute a rare event in immunocompetent patients. Most primary infections are asymptomatic, or the symptoms are acute and affect only the lungs. Oral lesions show a varied clinical appearance and are rarely painful. Mostly they presented as an ulcerative lesion with an elevated border, located mainly on the gingiva, tongue and palate. The reported cases presented with painful ulcers in gingivae and palatal mucosae with dysphagia and poor general health.

Oral lesions of histoplasmosis occurred only as secondary local manifestations of pulmonary or disseminated disease ${ }^{9}$. Some investigators have questioned this, claiming that primary oral histoplasmosis is possible and may develop as a result of direct inoculation of the fungus into the mucosa. Our first reported case did not present any pulmonary infection or disease involvement and second reported case had history of pulmonary tuberculosis with irregular ani-TB therapy. Clinicians usually diagnose primary oral histoplasmosis after discovering the lesions in the upper digestive tract in the absence of pulmonary signs and the diagnosis is based on identification of the fungus. For superficial and accessible oral lesions, cytologic evaluation and a biopsy usually are recommended. However, in cases in which the lesions are not accessible, serologic examination to detect antibodies or antigens may be a complementary diagnostic tool. Traumatic ulcers, necrotizing ulcerative gingivitis or stomatitis, syphilis, tuberculosis, squamous cell carcinoma and other mycoses, such as paracoccidioidomycosis should be considered as differential diagnosis. The reported cases were confirmed by histopathology with PAS staining. HIV1 and HIV2 were also excluded in both cases.

Though spontaneous remission of oral histoplasmosis is reported, failure to treat the infection in patients with AIDS may result in death. Hidden immunosuppression should be considered always especially in HIV infection. General practitioners must exercise caution when examining patients, because oral lesions of histoplasmosis may be the only sign of AIDS. The basic inflammatory response in a deep fungal infection is granulomatous, with macrophages and multinucleated giant cells dominating the histologic picture. Diffuse, small granulomas contain a variable number of yeastlike H. capsulatum cells, which are highlighted by methenamine silver and PAS staining ${ }^{1}$.

Histoplasmosis as systemic disease is treated by intravenous amphotericin B, which should be administered in a hospital because of its varied adverse effects, such as nausea, chills, fever, vomiting and renal complications. On the other hand, new and less toxic antifungal agents are available, such as the oral azoles, which are the drugs of choice for long-term maintenance therapy. Negroni and colleagues reported that treatment with itraconazole was favorable for localized oral lesions, when no systemic signs or symptoms could be detected. This drug appears to be safe and effective for both induction and maintenance therapies, with less toxicity than that of amphotericin B. Similarly our reported cases were treated by oral itraconazole with maintenance therapy. The disease responded well with regaining normal oral mucosa form and function and also improvement of general health.

\section{Conclusion:}

Histoplasmosis may affect immunocompetent as well as immunodeficient individuals, knowledge of the oral manifestations of histoplasmosis might enable clinicians to make earlier diagnoses and initiate therapy more quickly. It is important for general dental and medical practitioners to perform thorough clinical examinations and include histoplasmosis in the differential diagnosis of any persistent ulcerated oral lesions of both immunodeficient and immunocompetent patients.

\section{References:}

1. Wheat LJ. Laboratory diagnosis of histoplasmosis: update 2000. Semin Respir Infect-2001; 16(2):131-140.

2. Durkin MM, Connolly PA, Karimi K, et al. Pathogenic differences between North American and Latin American strains of Histoplasma capsulatum var. capsulatum in experimentally infected mice. J Clin Microbiol 2004; 42(9):4370-4373.

3. Islam N, Chowdhury NA. Histoplasmosis from Bangladesh: a case report. Bangladesh Med Res Counc Bull1982;8(1):21-24

4. Rappo U, Beitler UR, Faulhaber JR, et al. Expanding the horizons of histoplasmosis: disseminated histoplasmosis in a 
renal transplant patient aftera trip to Bangladesh. Transpl Infect Dis 2009; 1-6.

5. Mahbub MS, Ahsan MN, Miah MT, Alam MB, Gupta RD, Arif KM, Hasnain M: Disseminated histoplasmosis. J Medicine 2010; 11:70-73.

6. Rahman MM, Hossain SM, Faiz MA, Rahman J, Sultan MT, Dewanjee AK: Disseminated Histoplanmosis in AIDS. J.BCPS 2005; 23: 43-45.

7. Borges AS, Ferreira MS, Silvestre MT, Nishioka Sde A, Rocha A. Histoplasmosis in immunodepressed patients: study of 18 cases seen in Uberlândia, MG [in Portuguese]. Rev Soc Bras Med Trop 1997; 30(2): 119-124.

8. Wheat LJ, Kauffman CA. Histoplasmosis. Infect Dis Clin North Am 2003; 17(1):1-19.

9. Miller RL, Gould AR, Skolnick JL, Epstein WM. Localized oral histoplasmosis: a regional manifestation of mild chronic disseminated histoplasmosis. Oral Surg Oral Med Oral Pathol 1982; 53(4):367-374..

10. Loh FC, Yeo JF, Tan WC, Kumarasinghe G. Histoplasmosis presenting as hyperplastic gingival lesion. J Oral Pathol Med 1989; 18: 533-6.

11. Reddy P, Gorelick DF, Brasher CA, Larsh H. Progressive disseminated histoplasmosis as seen in adults. Am J Med 1970; 48: 629-36.
12. Cano MV, Hajjeh RA. The epidemiology of histoplasmosis: a review. Semin Respir Infect-2001; 16(2):109-118.

13. Rocha MM, Severo LC. Histoplasmose disseminada em pacientes com síndrome da imunodeficiência adquirida (SIDA): estudo de 25 casos [Disseminated histoplasmosis in patients with acquired immunodeficiency syndrome (AIDS): study of 25 cases.]. Rev Inst Med Trop São Paulo 1994;36(2):167-170.

14. Bullock WE. Histoplasma capsulatum. In: Mandell GL, Bennett JE, Dolin R, eds. Principles and practice of infectious diseases, 4th edn. New York: Churchill Living-stone, 1995; $2340-53$

15. Kirchner JT. Opportunistic fungal infections in patients with HIV disease: combating cryptococcosis and histoplasmosis. Postgraduate Med 1996; 99: 209-16.

16. Limaye AP, Connolly PA, Sagar M, et al. Brief Report: Transmission of Histoplasma capsulatum by organ transplantation. N Engl J Med 2000; 343: 1163-66.

17. Boutros HH, van Winckle RB, Evans GA, Wasan SM. Oral histoplasmosis masquerading as an invasive carcinoma. J Oral Maxillofac Surg 1995; 53: 1110-4.

18. Cobb CM, Shultz RE, Brewer JH, Dunlap CL. Chronic pulmonary histoplasmosis with an oral lesion. Oral Surg Oral Med Oral Pathol 1989; 67: 73-6. 